\title{
Spreading of lithium on a stainless steel surface at room temperature
}

\author{
C. H. Skinner ${ }^{\mathrm{a}}$, A.M. Capece ${ }^{\mathrm{a}}$, J.P. Roszell ${ }^{\mathrm{b}}$, B. E. Koel ${ }^{\mathrm{b}}$. \\ ${ }^{a}$ Princeton Plasma Physics Laboratory, Princeton NJ, 08543 USA \\ ${ }^{b}$ Department of Chemical and Biological Engineering, Princeton University NJ 08540 USA
}

\begin{abstract}
:
Lithium conditioned plasma facing surfaces have lowered recycling and enhanced plasma performance on many fusion devices and liquid lithium plasma facing components are under consideration for future machines. A key factor in the performance of liquid lithium components is the wetting by lithium of its container. We have observed the surface spreading of lithium from a mm-scale particle to adjacent stainless steel surfaces using a scanning Auger microprobe that has elemental discrimination. The spreading of lithium occurred at room temperature (when lithium is a solid) from one location at a speed of $0.62 \mu \mathrm{m} /$ day under ultrahigh vacuum conditions. Separate experiments using temperature programmed desorption (TPD) investigated bonding energetics between monolayer-scale films of lithium and stainless steel. While multilayer lithium desorption from stainless steel begins to occur just above $500 \mathrm{~K}\left(E_{\mathrm{des}}=1.54\right.$ $\mathrm{eV})$, sub-monolayer Li desorption occurred in a TPD peak at $942 \mathrm{~K}\left(E_{\mathrm{des}}=2.52 \mathrm{eV}\right)$ indicating more energetically favorable lithium-stainless steel bonding (in the absence of an oxidation layer) than lithium-lithium bonding.
\end{abstract}

PSI keywords: $\quad$ Lithium, Stainless steel, Surface Analysis

JNM keywords: P0500 Plasma-Materials Interaction, L0300 Lithium, S1300 Surface Effects, R0900 Redeposition.

PACS codes: 52.40.Hf Plasma-material interactions; boundary layer effects, $52.90 .+z$ Other topics in physics of plasmas and electric discharges

Corresponding author address:

Charles H. Skinner, Princeton Plasma Physics Laboratory, POB 451, Princeton NJ 08543 USA

Email: cskinner@pppl.gov, Phone: 609243 2214, Fax: 6092432665 


\section{Introduction}

Liquid plasma facing materials avoid the serious issues with radiation damage, helium blisters, thermal fatigue, and erosion lifetime that affect solid plasma facing components (PFCs) in a fusion reactor. Though less developed than solid plasma facing components, liquid PFCs enable the optimization of a liquid material for high particle flux and heat loads and the independent optimization of the solid, containing material for neutron loading[1]. Liquid lithium has the further advantage of binding with hydrogen isotopes, and lithium conditioning has reduced recycling and enhanced plasma performance on many fusion devices. Lithiumization of carbon plasma-facing components led to substantial advances in plasma performance in TFTR[2]. These were followed by experiments with a liquid Li capillary pore system at T11-M[3] and FTU[4], with a liquid Li tray in CDX-U[5] and with lithiumization of the TJ-II stellerator[6]. Also, a new liquid Li tokamak (LTX) began operation in 2010[7]. Lithiumization of ATJ graphite plasma facing tiles in the National Spherical Torus Experiment (NSTX) has shown strong beneficial effects such as improved confinement and reduction and elimination of ELMs[8,9]. A recent overview of lithium applications for fusion devices is given in ref. [10].

More broadly, the spreading of liquid metals is of high technological importance in areas such as soldering, brazing, and microelectronic fabrication, however the fundamental surface chemistry of the spreading of reactive liquids is not well established[11]. The contact angle at the liquid/solid interface has traditionally been used as a measure of wettability and interpreted in terms of thermodynamic quantities such as the far-field interfacial energy[12]. Surface energies control which of the three modes of equilibrium film growth occur on surfaces: (i) island or Volmer-Weber, (ii) layer plus island or Stranski-Krastanov, and (iii) layer by layer or Frank-van der Merwe [13]. At the atomic scale, surface energies and film growth modes depend on the relative strength of chemical bonding between atoms of the liquid compared to the bonding strength between atoms of the liquid and the solid substrate.

Capillary wicking of molten lithium on laser-textured 316L stainless steel and a TZM alloy at $866 \mathrm{~K}$ was observed at speeds up to $1.2 \mathrm{~cm} / \mathrm{s}$ [14]. Lithium films easily react with oxygen and water vapor[15] and the lithium oxide was reported to inhibit wetting of 316L stainless steel at temperatures up to $723 \mathrm{~K}$ [16]. A 1978 lithium literature review[17] reported "Purified lithium 
reportedly will not wet stainless steel at $315{ }^{\circ} \mathrm{C}[588 \mathrm{~K}]$ but does at $400{ }^{\circ} \mathrm{C}[673 \mathrm{~K}]$. Impure lithium will not wet stainless steel at temperatures below $482^{\circ} \mathrm{C}[755 \mathrm{~K}]$." [18]. The temperature dependence of the contact angle of liquid lithium on stainless steel, Mo, TZM alloy, Ta, and W has been studied and a minimum temperature of $588 \mathrm{~K}$ was reported to be necessary for wetting. [19]. Cleaning the surface with an argon glow discharge lowered this temperature to $570 \mathrm{~K}$ indicating the influence of surface contaminants and topology on the wetting process.

Diffusion of lithium on a Mo(112) single crystal surface was studied by a contact potential technique[20]. Data on the diffusion of Li out of an initial deposit 4.6-monolayers thick showed an extended flattened region at coverages above a monolayer $(1.1<\theta<2.5)$ that was consistent with an "unrolling carpet" model [21]. A related, detailed investigation of the emission of Sn from $\mathrm{Sn}$ islands on an Al substrate was performed using Atomic Force Microscopy (AFM) and scanning Auger microscopy (SAM)[22]. AFM images showed Stranski-Krastanov film growth with Sn islands bounded by crystallographic surfaces. After removal of the surrounding monatomic Sn layer by $\mathrm{Ar}^{+}$sputter cleaning, the reforming of this layer by a solid state wetting process was observed by repeating Auger linescans. The rate of spreading of Sn was measured to be $0.2-0.6 \mu \mathrm{m}^{2} / \mathrm{s}$. The spreading was found to be inhibited by impurities such as carbon or oxygen and at well-defined smooth crystallographic interfaces. SAM imaging should also be suitable for lithium spreading studies, and SAM imaging of a partially oxidized lithium surface has been demonstrated [23].

\section{Lithium - stainless steel chemical bonding}

Lithium coatings on a 316 stainless steel sample were studied using temperature programmed desorption (TPD) and Auger electron spectroscopy (AES) in a versatile UHV system containing several complementary surface analysis probes[24] that was operated at a base pressure of $2 \times 10^{-9}$ Torr. The stainless steel sample was cleaned of sulfur and phosphorus impurities using 1-keV $\mathrm{Ar}^{+}$ion sputtering, and oxygen and carbon were removed by heating to $1100 \mathrm{~K}$. Some phosphorus contamination remained at the surface as measured by AES $(<5$ at.\%). Oxygen and carbon contamination were less than 10 and 5 at.\%, respectively. Lithium was deposited onto the stainless steel sample by thermal evaporation from a SAES Getters alkali metal dispenser [25]. TPD was conducted by resistively heating the sample using a $10 \mathrm{~K} / \mathrm{s}$ linear temperature ramp and 
monitoring the desorbed Li with a UTI 100C quadrupole mass spectrometer (QMS) with the ionizer in direct line-of-sight of the sample. Temperature control was implemented using a Eurotherm 3508 PID controller, and the temperature was monitored using a type $\mathrm{C}$ thermocouple spot-welded to the back of the sample.

Figure 1 shows the Li (7 amu) QMS signal as a function of temperature during TPD of lithium films of varying thickness. The release of lithium from SAES Li dispenser is dependent on time and temperature and the temperature is controlled by the amount of current passed through the dispenser. The Li dispenser was operated at $7.1 \mathrm{~A}$ for $15 \mathrm{~s}$ (curve a), $60 \mathrm{~s}$ (curve b), $120 \mathrm{~s}$ (curve c), and $180 \mathrm{~s}$ (curves $\mathrm{d}$ and e). For curves (c) and (d) the sample was pre- flash heated to 550 and $525 \mathrm{~K}$, respectively. After each TPD experiment, the cleaning process was repeated and the next dose of lithium applied. The smallest Li dose produced a sub-monolayer film and had a $\mathrm{Li}$ desorption peak at $942 \mathrm{~K}$ (corresponding to a desorption activation energy, $E_{\text {des, }}$ of $2.52 \mathrm{eV} \mathrm{[26]).}$ With increasing Li dose, additional peaks at $772 \mathrm{~K}(2.05 \mathrm{eV})$ and $632 \mathrm{~K}(1.67 \mathrm{eV})$ emerged. These rather narrow Li TPD peaks are thought to be due to $\mathrm{Li}$ desorption from the thermal decomposition of lithium compounds (e.g. oxides) formed from reaction with contaminants present at the stainless steel surface and/or grain boundaries. After larger Li doses, a low temperature peak appeared, at $585 \mathrm{~K}$ for curve (e), which is due to the onset of desorption from a Li multilayer film, where Li-Li bonding controls the energetics. This region of the TPD curve was fit to an Arrhenius expression to give $E_{\mathrm{des}}=1.54 \mathrm{eV}$, which is close to the cohesive energy of metallic Li at $1.69 \mathrm{eV}[27]$.

The desorption activation energy is equal to the adsorption energy in those cases where there is no activation energy of adsorption, as in this case. Thus, for lithium on unoxidized stainless steel,

the $\mathrm{Li}$ adsorption energy $(2.52 \mathrm{eV}$ ) is much higher than the lithium-lithium adsorption energy (or binding energy) of $1.54 \mathrm{eV}$. In the absence of extensive surface oxidation, surface diffusion of lithium over stainless steel is energetically favorable, and a Stranski-Krastanov or Frank-van der Merwe growth mode is expected in the absence of kinetic limitations.

\section{Experimental setup.}

The spreading of lithium on stainless steel was observed via scanning electron microscopy (SEM) and elemental imaging using scanning Auger microscopy (SAM) in a Thermo Scientific 
Microlab 310 F Scanning Auger Microprobe and Microanalysis instrument. A standard SAM stub was fabricated from 316 stainless steel with a $0.8-\mathrm{mm}$ dia., $0.5-\mathrm{mm}$ deep hole at the center and prepared for vacuum using ultrasonic cleaning and a mild bakeout. Lithium was prepared in an argon glove box with trace impurity levels of $<0.1 \mathrm{ppm} \mathrm{O}_{2}$ and $6 \mathrm{ppm} \mathrm{H}_{2} \mathrm{O}$. A 12-mm dia. lithium rod (99.90 wt\% Li, FMC Corporation) was cleaved with a sharp stainless steel knife blade and a mm-sized Li particle was picked from the freshly exposed surface and pressed into the hole in the stub as shown in Fig. 2. The surface was then covered by an enclosure with an Oring seal that trapped the argon atmosphere and the stub was transferred to the loading chamber of the SAM with only a few seconds exposure to ambient air. This loading date is hereafter referenced as "day 0 ".

The SAM is equipped with a Schottky-type field emission electron source that was operated at $10 \mathrm{kV}$ and produced a current of $10-100 \mathrm{nA}$ on the sample. A SEM image of a corner of the lithium particle is shown in Fig. 3. Besides the collection of secondary electrons to form a standard SEM image, the SAM is equipped with a $150-\mathrm{mm}$ radius hemispherical electron analyzer with a resolution of $2 \%$ of the pass energy for Auger electron spectroscopy (AES). The analyzed electrons are detected by an array of 5 channeltrons. For these experiments the SAM stage was tilted $30^{\circ}$ from horizontal to orient the surface normal between the electron beam and the axis of the electron analyzer collection lens.

$\mathrm{An} \mathrm{Ar}^{+}$ion beam can be scanned over the sample for surface cleaning or etching to create a depth profile. The ion source was typically operated at $3 \mathrm{kV}$ and $1-2 \mu \mathrm{A}$ ion current. The ion beam spot on the sample was co-aligned with the electron beam spot by using a TV camera to observe optical emission from both beams striking a phosphor-coated stub. The total ion beam current incident on the sample was measured electrically with a $+90 \mathrm{~V}$ bias to the sample. The etching rate was measured by etching through a $100-\mathrm{nm}$ thick $\mathrm{Ta}_{2} \mathrm{O}_{5}$ sputter standard [28] and monitoring the $\mathrm{O}$ KLL and Ta MNN Auger lines as a function of ion current fluence ( $\left.\mu \mathrm{A}^{*} \mathrm{~min}\right)$.

\section{Scanning Auger imaging.}

An AES survey spectrum including both lithium and stainless areas exhibited the expected $\mathrm{Li}, \mathrm{O}$, $\mathrm{Cr}, \mathrm{Fe}$ AES peaks (Fig. 4). The absence of a carbon peak at $272 \mathrm{eV}$ indicates carbon is not a significant surface impurity $(<1 \%)$. Lithium Auger peaks are in the 40 to $\sim 50 \mathrm{eV}$ region, with a 
large chemical shift separating the lithium metal peak at $51.7 \mathrm{eV}$ from the lithium oxide peak at $40 \mathrm{eV}$ [29], with the cited peak energies denoting the sharp negative-going peaks in $\mathrm{dN} / \mathrm{dE}$ spectra. The metallic lithium Auger transition occurs by an interatomic KVV' process, where the prime denotes a neighboring site (there cannot be more than one valence hole on the same lithium atom). There is also potential interference from an iron-oxide related peak at $52 \mathrm{eV} \mathrm{[30]}$ near the metallic lithium peak. For these experiments the SAM base vacuum was $4-8 \times 10^{-7} \mathrm{~Pa}$ (2$6 \times 10^{-9}$ torr). Water vapor in the residual vacuum has a sticking coefficient on lithium of unity [15] and the surface of metallic lithium in the above vacuum conditions could be oxidized on a time scale of $15 \mathrm{~min}$. To avoid issues with the overlapping iron oxide Auger peak and timedependent lithium oxidation, SAM images were acquired using Li Auger electrons from lithium oxide in the $40-44 \mathrm{eV}$ region. A $256 \times 256$ pixel SAM image with good signal/noise could be acquired in $34 \mathrm{~min}$.

AES spectra in the $35-70 \mathrm{eV}$ region is shown in Fig. 5 for several spatial points on the SEM image. The curve labeled 'Li bulk' was from the lithium particle; the 'SS' curves were from two stainless steel regions, and the curve labeled 'Li prom.' is from a Li promontory region described in the next section. The peaks located at $41.4 \mathrm{eV}$ (with negative peaks at $43.4 \mathrm{eV}$ in a dN/dE plot) in both $\mathrm{Li}$ curves are assigned to $\mathrm{Li}-\mathrm{O}$ and were used for SAM imaging. This peak was present on the particle and promontory but not on the stainless steel surface The energy windows of the individual five channeltron (CEM) detectors are shown and two regions are labeled 'CEM peak' and 'CEM Bgnd' and correspond to separate images acquired from the total electron signals measured in these respective energy ranges. The resultant $\mathrm{Li}-\mathrm{O}$ image was then calculated using Eqn. 1:

$$
\text { Li-O image intensity }=\mathrm{C}_{1}+\mathrm{C}_{2} *[((\mathrm{Li}-\mathrm{O} \text { peak })-(\text { Bgnd })) /(\text { Bgnd })]
$$

where $\mathrm{C}_{1}$ is a constant used to avoid negative intensities and $\mathrm{C}_{2}$ is a constant used to scale the image to an 8 -bit range. The image contrast was enhanced by adjusting the image levels to encompass most of the range of 256 levels. 


\section{Surface spreading of lithium on stainless steel.}

On the $2^{\text {nd }}$ day after the lithium particle was mounted on the stainless steel stub (day 2) the $\mathrm{Ar}^{+}$ beam was used to etch the surface. The etch depth was estimated to be $106 \mathrm{~nm}$ by scaling the ion beam fluence used with that needed to sputter through the $100 \mathrm{~nm} \mathrm{Ta}_{2} \mathrm{O}_{5}$ sputter standard. Ref.[31] lists the 3-keV Ar ${ }^{+}$sputter rates for $\mathrm{Ta}_{2} \mathrm{O}_{5}\left(0.143 \mathrm{~nm} \mathrm{~min}^{-1} \mu \mathrm{A}^{-1} \mathrm{~cm}^{-2}\right)$ and $\mathrm{Fe}_{3} \mathrm{O}_{4}(0.148$ $\mathrm{nm} \mathrm{min}^{-1} \mu \mathrm{A}^{-1} \mathrm{~cm}^{-2}$ ), and values for other $\mathrm{Fe}$ and $\mathrm{Cr}$ oxides are within $50 \%$. The etch depth is simply expressed as the $\mathrm{Ta}_{2} \mathrm{O}_{5}$ equivalent. The etching was repeated on day 9 with $72 \mathrm{~nm}$ removed and on day 24 with $14 \mathrm{~nm}$ removed. $\mathrm{Ar}^{+}$sputtering of stainless steel is known to remove impurity overlayers and roughen the surface topography [32], and similar effects occur for lithium. On day 15 an interesting feature resembling a 'tide mark' was observed in an SEM image of a corner of the particle. This feature was then imaged using Li-O Auger electrons to produce a SAM chemical map and is shown in Fig. 6(a). A 'promontory' was observed to extend from the corner of the lithium particle and appears to have a smooth surface, distinct from the topography of the lithium particle and from the machining marks in the stainless steel surface. This Li-O SAM image was repeated on day 17 and remarkably, the size of the Li-O promontory was noticeably larger. Subsequent Li-O SAM images on days 18, 21, 23, 24 and 44 showed that the lithium had continued to spread out on the stainless steel. A sequence of SAM Li-O images on days 15, 21 and 44 are shown in Fig. 6 (a-c). Some bands parallel to the propagation front are apparent in the promontory image, together with some smaller extrusions. The SAM magnification in the $\mathrm{x}$ and $\mathrm{y}$ directions in the image was calibrated with a 400-mesh copper grid that was in turn referenced to an optical standard. The distance that the lithium had spread was measured from the digital image along the direction shown by the white scale superimposed on the SAM images in Fig. 6 and the average of three measurements is plotted in Fig. 7. Eqn. 2 shows a linear regression fit to the points for days $15,17,18,21,23,24$, which had a correlation coefficient of $\mathrm{R}^{2}=0.97$,

$$
d=0.62 t-4.5
$$

Eqn. 2

where $d$ is the distance $(\mu \mathrm{m})$ and $t$ is the time (days). Rather than a square root dependence typical of a diffusive process, the promontory expanded at a constant speed of $0.62 \mu \mathrm{m} / \mathrm{day}$ between days 15 and 24. This trend line intercepts the $x$-axis at day 7.3, close in time to the day 9 
etch. Between day 24 and the final point on day 44 this speed was reduced to $0.18 \mu \mathrm{m} /$ day, possibly as a result of the third etch on day 24 .

The Fe LMM Auger peaks that appear as a triplet at 598, 651, and $703 \mathrm{eV}$ were observed on the stainless surface, but not on the promontory. A trace of these Fe lines was apparent on the $\mathrm{Li}$ particle, possibly from contamination by the stainless knife used to prepare the Li particle. The absence of the Fe lines on the promontory may be used to estimate a minimum thickness of the spreading Li layer. The AES probing depth can be given as three times the inelastic mean free path $(\lambda)$ for the Fe LMM $703 \mathrm{eV}$ Auger electrons, which can be calculated to be $\lambda=3.1 \mathrm{~nm}$ for metallic $\mathrm{Li}$ and $\lambda=2.1 \mathrm{~nm}$ for $\mathrm{Li}_{2} \mathrm{O}$ [33]. From this we conclude that the thickness of the promontory is more than $6 \mathrm{~nm}$.

\section{Summary.}

We have observed surface spreading of solid lithium onto stainless steel at room temperature. While lithium is known to wet stainless steel at elevated temperatures, this is the first report of lithium reactive wetting and diffusion at $300 \mathrm{~K}$. This occurred at the corner of a small lithium particle where evidently there is a feature that nucleated this growth. The spreading appeared to be associated with etching of the lithium and stainless surfaces by an $\mathrm{Ar}^{+}$ion beam. The rate of lithium spreading was $0.62 \mu \mathrm{m} /$ day between days 15 and 24 after the lithium particle was pressed onto the stainless surface, decreasing to $0.18 \mu \mathrm{m} / \mathrm{d}$ between days 24 and 44 . The thickness of the spreading lithium layer was estimated to be at least $6 \mathrm{~nm}$.

The low thermal desorption energy for Li sublimation from thick Li films and much larger energy for thermal desorption of Li from the Li monolayer on stainless steel indicates that it can be energetically favorable for lithium to spread over a reduced metallic stainless surface. However, this behavior was not universal, and our observations were made from a single promontory originating from one corner of a lithium particle. The spreading was correlated with $\mathrm{Ar}^{+}$ion beam etching, that removed contamination and oxide films from the surface. Future work will seek to identify the conditions for nucleation and spreading in order to facilitate wetting in future liquid metal PFCs. We also plan to investigate lithium spreading as a function of surface temperature on stainless and other PFC materials such as molybdenum and tungsten. 


\section{Acknowledgements.}

The authors thank D. Labrie, G. Smalley and A. Plasencia for technical assistance. Support was provided by the U.S. DOE Contract Nos. DE AC02-09CH11466 and DE-SC0008598. BEK acknowledges support by the U.S. DOE under Award Number DE-SC0008598.

\section{Figure Captions:}

Fig. 1 TPD spectra of lithium (7 amu) desorption measured by a mass spectrometer from lithium films on a stainless substrate (color on-line). The amount of lithium prior to TPD measurements increases from (a) to (e) - see text.

Fig. 2 Photograph of a particle of lithium pressed onto a 13-mm diameter stainless steel SAM stub.

Fig. 3 SEM image of corner of lithium particle (left) on stainless stub surface. The curved tracks on the right are machining marks on the stainless surface. The image covers 304 microns horizontally.

Fig. 4 AES survey spectrum of lithium and stainless surface.

Fig. 5 Multipoint AES spectra of lithium bulk, three locations on the lithium promontory, and two locations on the stainless steel surface. The channeltron (CEM) energies used in SAM imaging are also shown.

Fig. 6 SAM images showing the spreading of lithium from the particle in the lower left over the stainless steel substrate. Panel (a) was taken $15 \mathrm{~d}$, panel (b) $21 \mathrm{~d}$, and panel (c) $44 \mathrm{~d}$ after the lithium was pressed into the stainless steel stub. The spreading along the path shown by the white scale bar was measured for every SAM image (some not shown here) and is plotted in Fig. 8. The scale bar is in microns

Fig. 7 Progressive spreading of Li as measured on the dashed track in Fig. 7. Day 0 refers to the day when the lithium particle was mounted on the stainless steel stub. Vertical lines mark days when $\mathrm{Ar}^{+}$beam etching was performed. 


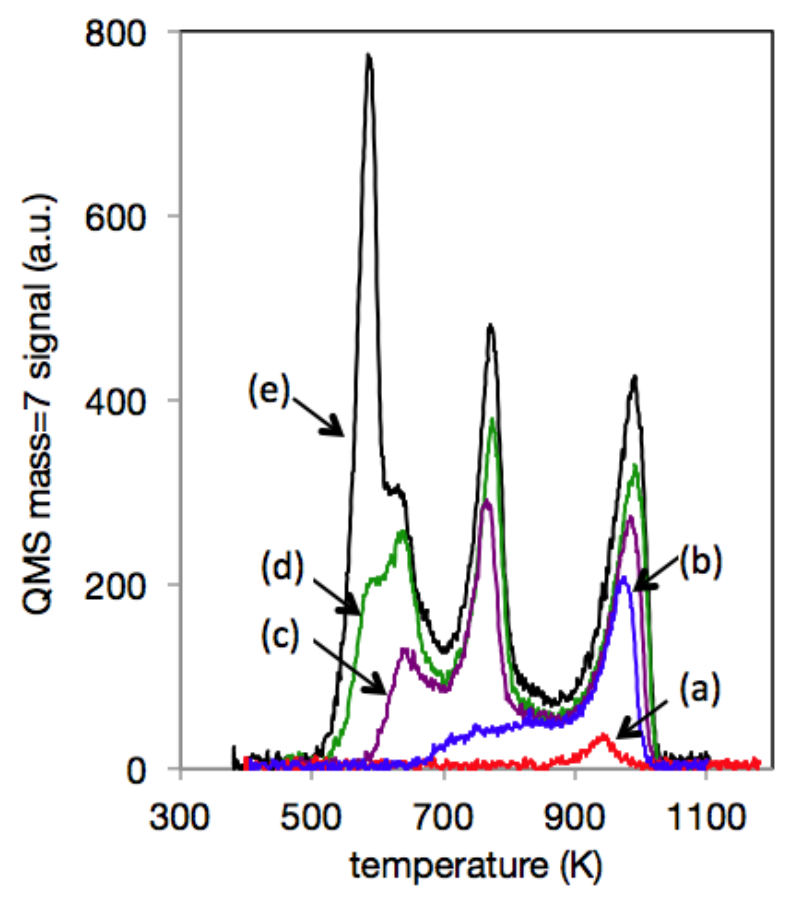

Fig. 1 TPD spectra of lithium ( $7 \mathrm{amu}$ ) desorption measured by a mass spectrometer from lithium films on a stainless substrate (color on-line). The amount of lithium prior to TPD measurements increases from (a) to (e) - see text. 


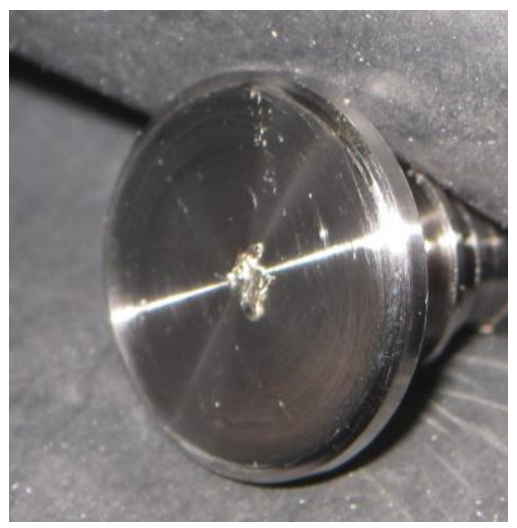

Fig. 2 Photograph of a particle of lithium pressed onto a $13 \mathrm{~mm}$ diameter stainless steel SAM stub. 


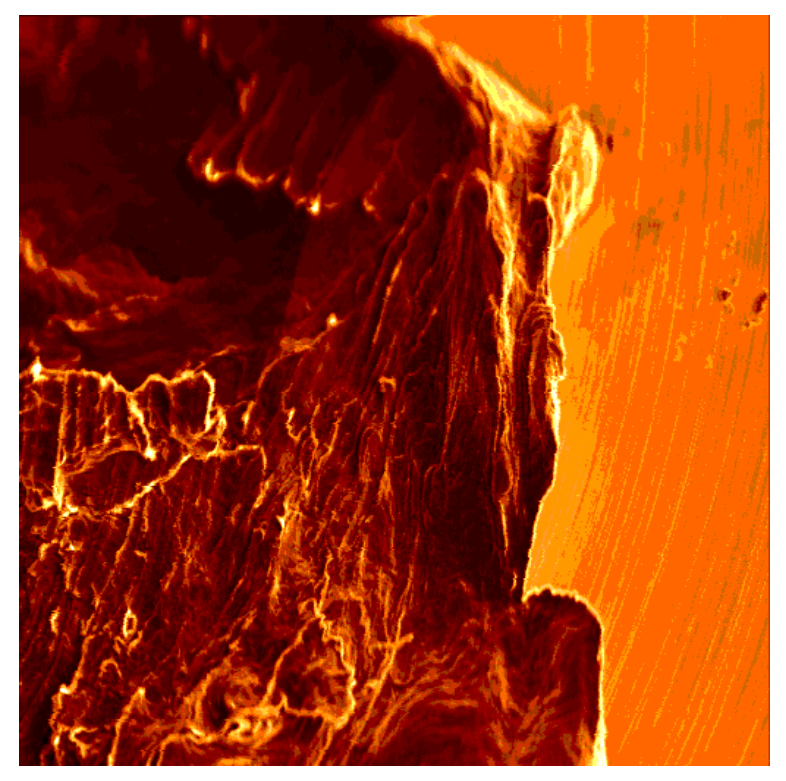

Fig. 3 SEM image of corner of lithium particle (left) on stainless stub surface. The curved tracks on the right are machining marks on the stainless surface, the scale bar is in microns. The image covers 304 microns horizontally. 


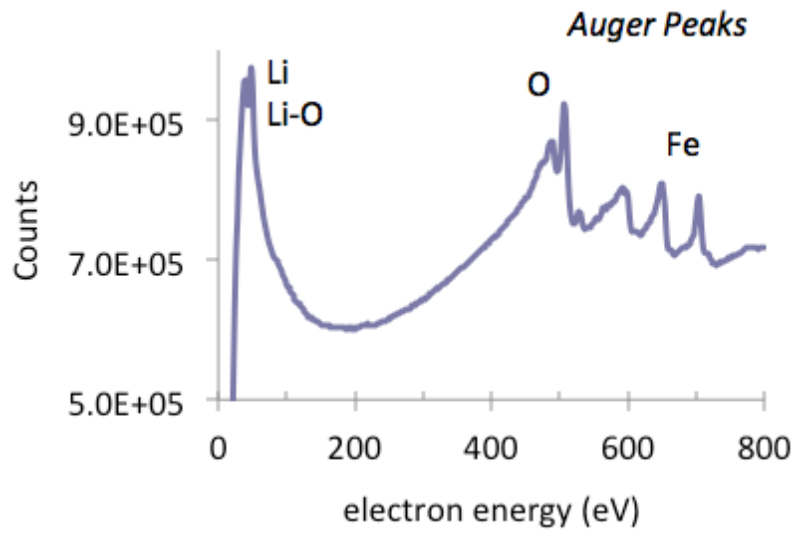

Fig. 4 AES survey spectrum of lithium and stainless surface. 


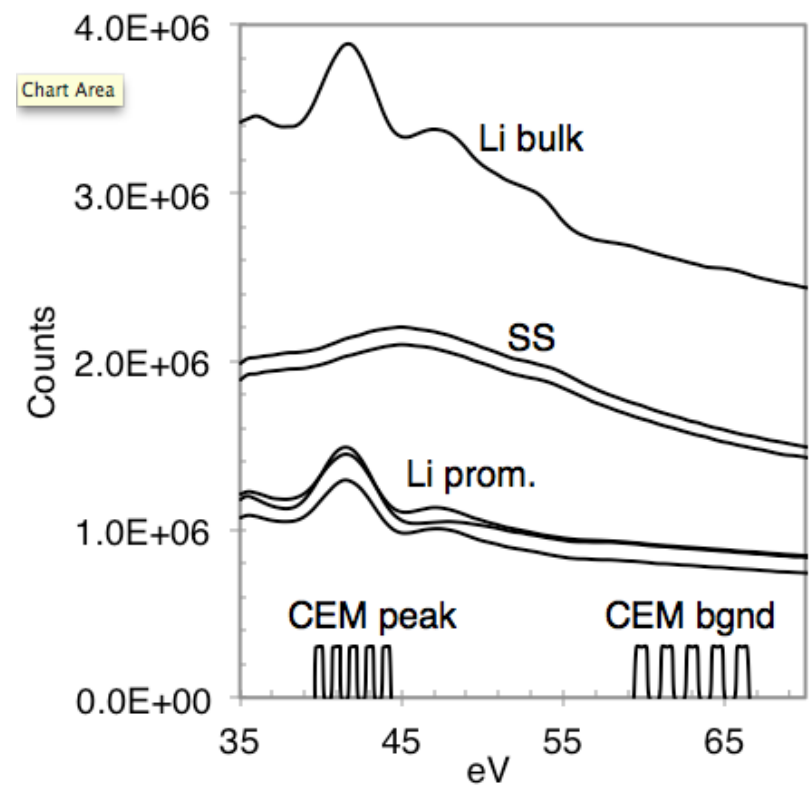

Fig. 5 Multipoint AES spectra of lithium bulk, three locations on the lithium promontory, and two locations on the stainless steel surface. The channeltron (CEM) energies used in SAM imaging are also shown. 
Fig. 6 SAM images showing the spreading of lithium from the particle in the lower left over the stainless steel substrate. Panel (a) was taken $15 \mathrm{~d}$, panel (b) $21 \mathrm{~d}$, and panel (c) $44 \mathrm{~d}$ after the lithium was pressed into the stainless steel stub. The spreading along the path shown by the white scale bar was measured for every SAM image (some not shown here) and is plotted in Fig. 8. The scale bar is in microns.

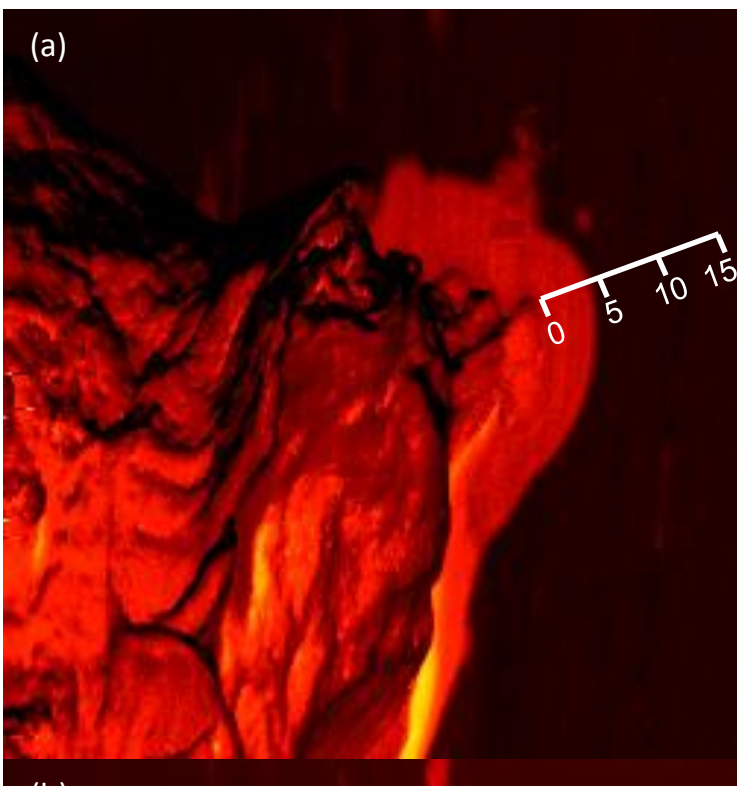

(b)

(c) 


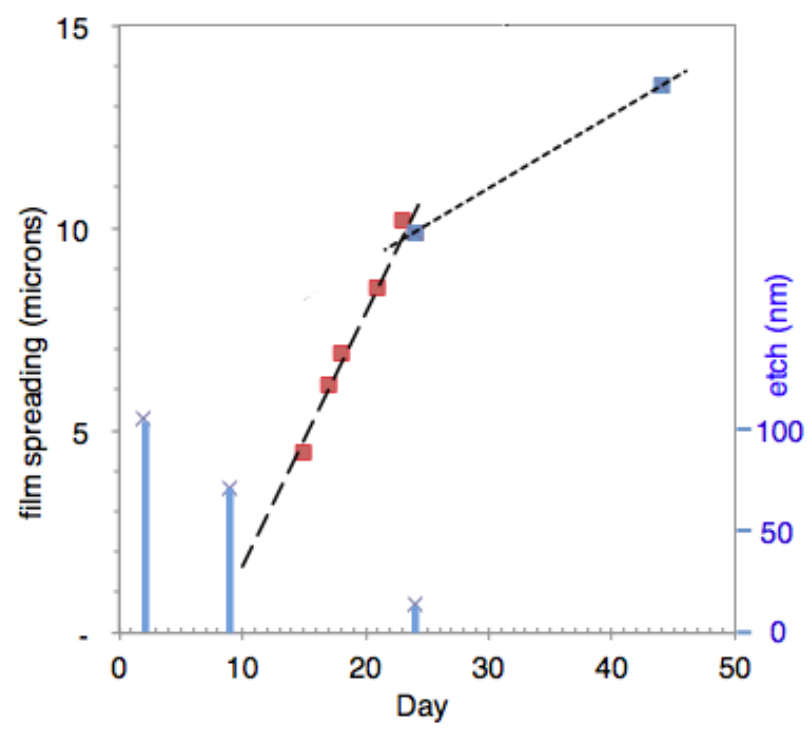

Fig. 7 Progressive spreading of $\mathrm{Li}$ as measured from the length of the dashed track in Fig. 7. Day 0 refers to the day when the lithium particle was mounted on the stainless steel stub. Sample etching by the Ar beam and the etch depths are shown by the vertical bars.

\section{References}

[1] J.W. Coenen et al., Phys. Scr. T159 (2014) 014037.

[2] D. K. Mansfield et al., Phys. Plasmas 3 (1996) 1892.

[3] S. V. Mirnov et al., Plasma Phys. Control. Fusion 48 (2006) 821.

[4] M. L. Apicella et al., Journal of Nuclear Materials 386-388 (2009) 821.

[5] R. Kaita et al., Phys. Plasmas 14 (2007) 056111.

[6] J. Sanchez et al., Nucl. Fus. 49 (2009) 104018.

[7] R. Kaita et al., Fusion Eng. Des. 85 (2010) 874.

[8] H. W. Kugel et al., J. Nucl. Mater. 390-391 (2009) 1000.

[9] M.G. Bell et al., Plasma Phys. Contr. Fusion 51 (2009) 124054.

[10] M. Ono et al., Nucl. Fusion 52 (2012) 037001.

[11] E. Saiz and A.P. Tomsia, Nature Materials 3 (2004) 903.

[12] P.G. de Gennes, Rev. Mod. Phys. 57 (1985) 827.

[13] J.A. Venables, G.D.T. Spiller, and M. Hanbüken, Rep. Prog. Phys., 47 (1984) 399. 
[14] T.F. Lin, T.A. Palmer, K.C. Meinert, N.R. Murray and R. Majeski, J. Nucl. Mater., 433 (2013) 55 .

[15] C.H. Skinner, R. Sullenberger, B.E. Koel., M.A. Jaworski, H.W. Kugel, J. Nucl. Mater., 438 (2013) S647.

[16] E. Platacis et al., Magnetohydrodynamics 48 (2012) 667.

[17] D.W. Jeppson, J.L. Ballif, W.W. Yuan, B.E. Chou. 'Lithium Literature Review: Lithium's Properties and Interactions’ p.26 Hanford Report HEDL-TME 78-15 UC-20 April 1978.

[18] J.O. Cowles and A. D. Pasternak, Lithium Properties Related to Use as a Nuclear Reactor Coolant. UCRL-50647, Lawrence Radiation Laboratory, CA, April 18, 1969.

[19] P. Fiflis, W. Xu, D. Andruczyk, D. Curelli and D.N. Ruzic, Fus. Eng. Des. (2014) in press.

[20] A.G. Naumovets, M.V. Paily and Yu.S. Vedula, Progress in Surf. Sci. 48 (1995) 59.

[21] R Gomer, R Wortman, and R Lundy, The Journal of Chemical Physics, 26, (1957). 11471164.

[22] E. Klein, B. Schwarz, C. Eisenmenger-Sittner, C Tomastik, P.B. Barna and A Kovács, Vacuum 80 (2005) 74.

[23] N. Ishida and D. Fujita, Journal of Electron Spectroscopy and Related Phenomena, 186 (2013) 39 .

[24] A. Sellidj, B.E. Koel, J. Phys. Chem. 97 (1993) 10076.

[25] SAES Getters USA, Inc. 1122 East Cheyenne Mountain Blvd. Colorado Springs, CO 80906, USA, www.saesgetters.com

[26] P. A. Redhead, Vacuum (1966) 203.

[27] W. Ching and J. Callaway, Phys. Rev. B 9 (1974) 5115.

[28] C. P. Hunt and M. P. Seah, Surface and Interface Analysis 5 (1983) 199.

[29] R.E. Clausing and D.S. Easton Surf. Sci., 36 (1973) 377.

[30] A.G. Sault, Appl. Surf. Sci. 74 (1994) 249. 
[31] H. Viefhaus, K. Hennesen, M. Lucas, E.M. Müller-Lorenz and H.J. Grabke, Surface and Interface Analysis 21 (1994) 665.

[32] R. Bastasz and G.J. Thomas, J. Nucl. Mater. 76 \& 77 (1978) 183.

[33] C. J. Powell and A. Jablonski, NIST Electron Inelastic-Mean-Free-Path Database Version 1.2, National Institute of Standards and Technology, Gaithersburg, MD (2010). 